\title{
Promoter methylation and histone modifications affect the expression of the exogenous DsRed gene in transgenic goats
}

\author{
M.T. Nuo, J.L. Yuan, W.L. Yang, X.Y. Gao, N. He, H. Liang, M. Cang and \\ D.J. Liu
}

Key Laboratory of Mammalian Reproductive Biology and Biotechnology of the Ministry of Education, Inner Mongolia University, Hohhot,

Inner Mongolia, China

Corresponding author: D.J. Liu

E-mail: nmliudongjun@sina.com

Genet. Mol. Res. 15 (3): gmr.15038560

Received February 17, 2016

Accepted April 15, 2016

Published August 29, 2016

DOI http://dx.doi.org/10.4238/gmr.15038560

Copyright (C) 2016 The Authors. This is an open-access article distributed under the terms of the Creative Commons Attribution ShareAlike (CC BY-SA) 4.0 License.

ABSTRACT. Transgene silencing, which is common in transgenic
plants and animals, limits the generation and application of genetically
modified organisms, and is associated with the exogenous gene copy
number, the methylation status of its promoters, and histone modification
abnormalities. Here, we analyzed the expression of the exogenous
gene DsRed and the methylation status of its cytomegalovirus (CMV)
promoter in six healthy transgenic cashmere goats and transgenic nuclear
donor cells. The CMV promoter exhibited high methylation levels (74.4-
$88.2 \%)$ in four of the goats, a moderate methylation level (58.7\%) in
one, and a low methylation level (21.2\%) in one, while the methylation
level of the transgenic nuclear donor cells was comparatively low
$(14.3 \%)$. DsRed expression was negatively correlated with promoter
methylation status. Transgenic cashmere goats carried one to three 
copies of the CMV promoter fragment and one to six copies of the DsRed fragment, but copy number showed no obvious correlation with DsRed expression. After treatment with the methylation inhibitor 5-azacytidine, DsRed expression in transgenic goat cells significantly increased and CMV promoter methylation significantly decreased; this indicated an inverse correlation between promoter methylation status and DsRed expression. After treatment with the histone deacetylase inhibitor trichostatin A, DsRed expression increased, indicating that an abnormal histone modification in transgenic goats is also involved in exogenous gene silencing. These findings indicate the potential of trichostatin A and 5-azacytidine to rescue the biological activity of silenced exogenous transgenes in adult-derived transgenic cells under culture conditions.

Key words: 5-Az; Histone modification; Promoter methylation; Transgenic goat; TSA

\section{INTRODUCTION}

Transgenic animal technology has undergone rapid development in recent years, and has been applied to the genetic modification and breeding of new species of livestock (Rudolph, 1999). However, the phenomenon of transgene silencing has restricted the effective expression of exogenous genes in transgenic animals, thus decreasing the breeding efficiency of new types of transgenic animals. Consequently, studies on the mechanism of transgene silencing and possible solutions have important practical significance.

As the strongest promoter in vitro, the cytomegalovirus (CMV) promoter is widely used in research on vector establishment and gene expression. However, there are large differences in the expressions of proteins driven by the CMV promoter between different types of cells cultured in vitro; for example, the CMV promoter can drive a strong expression of exogenous genes in 293T and cutaneous metastatic malignant tumor cells and differentiated neurons, while the expression of these genes in MRC5 and MSC cells is weak; this difference between somatic cells might be associated with promoter silencing (Teschendorf et al., 2002; Brooks et al., 2004). Furthermore, in U87 neurocytoma cells, green fluorescent protein (GFP) expression driven by the CMV promoter has been shown to be inhibited as the passage number increases, while treatment with the histone deacetylase inhibitor trichostatin A (TSA) or the methylation inhibitor 5-azacytidine (5-Az) rescues GFP expression. These observations suggest that CMV promoter-induced gene silencing is closely associated with DNA methylation and histone deacetylation (Grassi et al., 2003). In the genome of transgenic sheep generated using a lentivirus vector, the exogenous CMV promoter exhibited different levels of methylation that were associated with the suppression of exogenous gene expression (Hofmann et al., 2003).

Currently, there is a lack of research related to transgene expression at the epigenetic level in transgenic cashmere goats. Here, we conducted a systematic study in transgenic cashmere goats obtained by the somatic cell cloning method to investigate the mutual relationship between exogenous DsRed gene expression and the methylation status of the $\mathrm{CMV}$ promoter. Furthermore, we evaluated changes in exogenous gene expression in response to 5-Az and TSA treatment to determine the specific mechanism underlying the regulation of

Genetics and Molecular Research 15 (3): gmr.15038560 
exogenous gene expression. The results of the present study provide an experimental basis for improving the expression efficiency of exogenous genes in in vitro-cultured transgenic cells.

\section{MATERIAL AND METHODS}

\section{Ethics statement}

All of the study procedures adhered to the International Guiding Principles for Biomedical Research Involving Animals issued by the Council for the International Organizations of Medical Sciences, and were approved by the Institutional Animal Care and Use Committee of Inner Mongolia University. The study was also approved by the owner of YiWei White Cashmere Goat Farm (Ordos, China; 39¹2'6.71"N, 10755'1.30"E).

\section{Experimental animals and cell cultures}

The transgenic cells used in this experiment were collected from the ear tips of six healthy transgenic cashmere goats. The nuclear donor cells of the goats were transfected with eukaryotic expression vectors, as shown in Table 1, using Lipofectamine ${ }^{\mathrm{TM}} 2000$ (Life Technology, USA) according to instructions provided by the manufacturer. Resistant clones were obtained by G418 selection. Nuclear transfer into in vitro-matured oocytes was then performed as described previously (Guo et al., 2009). The reconstructed embryos were cultured for $48 \mathrm{~h}$ and the cleavage rate was calculated. Embryos with suitable morphologies were selected for transplantation into estrus-synchronized recipients. Approximately five embryos were transplanted into each recipient. Pregnancy was assumed based on the absence of estrus after two continuous estrus cycles (Yuan et al., 2012).

Table 1. Identity of transgenic cashmere goats.

\begin{tabular}{l|c|c|c|c|c|c|c|c}
\hline Transfection vectors & \multicolumn{2}{|c|}{ KKAP6-1-T $\beta 4-C M V p-D s R e d$} & pKAP6-1-VEGF-CMVp-DsRed & \multicolumn{3}{|c|}{ pKAP6-1-IGF1-CMVp-DsRed } & Empty \\
\hline Transgenic cashmere goats or cell No. & ZK110321 & ZK110324-2 & ZK110323-3 & ZK110324-1 & ZK0310-1 & ZK0311-1 & CFC3-KI & CFC3 \\
\hline Sex & Male & Male & Female & Female & Female & Female & Female & Female \\
\hline
\end{tabular}

All of the transgenic cashmere goats produced were the result of random integration. Transgenic nuclear donor cells and fibroblasts isolated from cashmere goat fetuses (wild type, after a 40-day gestation) transfected with an empty vector (named CFC3) (Guo et al., 2009) were used as controls (Table 1). The transgenic cashmere goats were generated by transfection with the pDsRed2-1 vector (Catalog No. 632405; Clontech, USA), which expresses the exogenous DsRed gene under the control of the CMV promoter from the pIRES2-EGFP vector (Catalog No. 6029-1; Clontech, USA). The pDsRed2-1 vector was ligated at multiple cloning sites with the epidermal specific keratin 6-1 promoter (pKAP6-1), thymosin $\beta 4$ (T $\beta 4$ ), and vascular endothelial growth factor 164 (VEGF) from the cashmere goat, or insulin-like growth factor I (IGF1) from sheep. All of these target genes were selected because they were believed to have the potential to accelerate hair follicle development and increase the production of cashmere wool (Harris et al., 1993; Yamamoto et al., 1997; Philp et al., 2004).

The cells were cultured in Dulbecco's modified eagle medium/nutrient mixture F-12 at a ratio of 1:1 (Catalog No. SH30023.018; HyClone, USA) supplemented with $10 \%$ standard fetal bovine serum (Catalog No. TBD31HB; TBD, Tianjin, China) and 1\%

Genetics and Molecular Research 15 (3): gmr.15038560 
penicillin/streptomycin solution (Catalog No. SV30010; HyClone). A solution of $1 \mathrm{X}$ trypsinethylenediaminetetraacetic acid (0.25\%) (Catalog No. 25200-056; Gibco, USA) was used for releasing adherent cells for passaging the cultured cells every 3-5 days (1:3 dilution).

\section{Fluorescence imaging of transgenic cashmere goats}

Images of red fluorescent protein expression in the goats were obtained in the dark using portable ultraviolet (UV) light equipment and a digital camera (EOS 600D; Canon, Japan).

\section{Polymerase chain reaction (PCR) analysis of transgenic cashmere goats}

The genomic DNA used in this experiment was extracted from fibroblasts (passage P5-P6) obtained from the ear tips of the goats using a DNeasy ${ }^{\circledR}$ Blood and Tissue Kit (Catalog No. 69504; Qiagen, Germany). Primers were then designed, including CMVp + DsRed + PolyA primer (Abbreviation for CDA Primer) based on the full-length sequence containing the DsRed expression cassette in the transfection vector after extraction of genomic DNA. PCR amplification was performed using a cashmere goat glyceraldehyde 3-phosphate dehydrogenase (GAPDH) fragment as an internal reference for the genomic sequence using PCR polymerase in a GoTaq ${ }^{\mathbb{B}}$ Green Master Mix (Catalog No. M7122; Promega, USA).

The amplified fragment was obtained by purification of the PCR products following agarose gel electrophoresis using a GeneJET ${ }^{\mathrm{TM}}$ Gel Extraction Kit (Catalog No. K0691; Thermo Scientific, USA). The fragment was then ligated overnight at $16^{\circ} \mathrm{C}$ using a pMD19-T-Vector Cloning Kit (Catalog No. 6011; TaKaRa, Japan), and transformed into DH5 $\alpha$-competent cells (Catalog No. CB101; Tiangen, China). Positive clones selected from the overnight cultures were sent to Invitrogen (Beijing, China) for sequencing.

\section{Absolute quantitative PCR (qPCR) analysis of the DsRed copy number in transgenic cashmere goats}

The standard vector for qPCR-based detection of the copy number of DsRed in the goats was obtained by ligation of the $C M V p+D s R e d+$ PolyA expression cassette fragment obtained during PCR identification into the pMD19-T-vector. A standard plasmid solution $(51 \mathrm{ng} / \mu \mathrm{L})$ containing $11,274,048,780$ copies $/ \mu \mathrm{L}$ was prepared, and the concentration was calculated according to the following formula: [concentration $(\mathrm{ng} / \mu \mathrm{L}) \times 6.02 \times 10^{23} /$ (base number x $\left.650 \times 10^{9}\right)$ ]. A 12-point standard curve of plasmid samples was prepared by 10 -fold serial dilution. Two pairs of primers were designed to amplify the sequence of the DsRed expression cassette: one pair corresponded to an internal sequence of the CMV promoter and one pair corresponded to an internal region of DsRed. Fluorescence qPCR was conducted using SYBR ${ }^{\circledR}$ Premix Ex Taq ${ }^{\mathrm{TM}}$ II (Tli RNAaseH Plus) (Catalog No. RR042A; Takara) in a 25$\mu \mathrm{L}$ volume using the Analytik Jena qTOWER 2.2 system. The absolute quantitative standard curve regression equations were as follows: primer $\mathrm{CMV}, \mathrm{Ct}=-2.964 \log$ (conc.) $+32.366\left(R^{2}\right.$ $=0.99379$, PCR efficiency $=1.17)$; primer DsRed, $\mathrm{Ct}=-3.2327 \log ($ conc $)+33.5815\left(R^{2}=\right.$ 0.99711 , PCR efficiency $=1.04$ ). Melting curve analysis was conducted for two reactions that exhibited single peaks, which indicated good reaction specificity.

Genomic DNA was extracted from fibroblasts obtained from the ear tips of the six goats and CFC3 fetal fibroblasts using a Wizard ${ }^{\circledR}$ Genomic DNA Purification Kit (Catalog

Genetics and Molecular Research 15 (3): gmr.15038560 
No. A1125; Promega). The extracted DNA was diluted to $51 \mathrm{ng} / \mu \mathrm{L}$ for real-time fluorescence qPCR amplification. The exogenous gene copy number of the corresponding samples was calculated by linear regression based on the standard curves obtained using the CMV and DsRed primer pairs. The exogenous gene copy number in the genome of a transgenic cashmere goat was calculated using the formula $\left(650 \times \mathrm{G} \mathrm{x} 10^{9} \times 2 \times \mathrm{N}\right) /\left(6.02 \times 10^{23}\right)$, where $\mathrm{G}(2.66 \times$ $10^{9} \mathrm{bp}$ ) represents the size of the cashmere goat genome, 650 is the molecular weight of DNA mononucleotides, $\mathrm{N}$ is the copy number of the CMV or DsRed fragment, and $6.02 \times 10^{23}$ is the Avogadro constant.

\section{5-Az and TSA treatment of fibroblasts from transgenic cashmere goats}

Fibroblasts obtained from the ear tips of the six goats and CFC3-KI cells (at passage P6) were cultured in the presence of a concentration gradient of 5-Az $(5,10,15,20$, and 25 $\mu \mathrm{M}$, Catalog No. A2385-250MG; Sigma-Aldrich, USA) or TSA $(0,0.25,0.5,0.75$, and $1 \mu \mathrm{M}$, Catalog No. T1952-200 UL; Sigma-Aldrich) for one week to determine the optimal treatment concentrations for each cell line. This was determined by qPCR analysis of the concentration required to induce the maximal increase in DsRed mRNA expression. The optimal treatment concentrations were 10 and $1 \mu \mathrm{M}$, respectively, for 5-Az and TSA.

The cell lines were then treated with 5-Az at the optimal concentration for 7 days by replacing the drug-containing culture medium every other day. Alternatively, cells were cultured to $90 \%$ confluence and treated with TSA for $24 \mathrm{~h}$, and $D$ sRed expression was analyzed by qPCR.

\section{Observation of adherent cells by fluorescence phase-contrast microscopy}

Red fluorescence expression in the treated cell lines was evaluated after one week by fluorescence phase-contrast microscopy (Zeiss, Germany), with fluorescence emission detected using an excitation device (HBO100) and recorded with the AxioVision Release 4.8.2 photographic software.

\section{qPCR analysis of DsRed expression}

DsRed expression was analyzed by qPCR. Total RNA was extracted from the cells at passage P6 using an RNeasy ${ }^{\circledR}$ Plus Mini Kit (Catalog No. 74134; Qiagen).

cDNA was generated by reverse transcription using a PrimeScript ${ }^{\mathrm{TM}}$ RT Reagent Kit. Genomic DNA was eliminated by gDNA Eraser (Perfect Real-Time) (Catalog No. RR047A; Takara). Real-time fluorescence qPCR was performed on an Analytik Jena qTOWER 2.2 fluorescence qPCR amplifier in a $25-\mu \mathrm{L}$ reaction volume with $\mathrm{SYBR}^{\circledR}$ Premix Ex Taq II (Tli RNAaseH Plus) (TaKaRa). Expression of the exogenous DsRed gene and the GAPDH housekeeping reference gene was analyzed using cDNA generated from CFC3-KI cells, and total RNA was used as a standard to verify the amplification efficiency of the two pairs of primers. A seven-point standard curve of the cDNA samples $(50 \mathrm{ng} / \mu \mathrm{L})$ from the CFC3-KI cells was prepared by five-fold serial dilution. The absolute quantitative standard curve regression equations were as follows: GAPDH primer, $\mathrm{Ct}=-3.38 \log$ (conc.) +17.79 $\left(R^{2}=0.99851\right.$, PCR efficiency $\left.=0.98\right)$; DsRed primer, $\mathrm{Ct}=-3.18 \log ($ conc. $)+17.05\left(R^{2}=\right.$ 0.99585 , PCR efficiency $=1.06$ ).

The following primers were used for qPCR analysis; for DsRed, forward: 5'-TGAAGG

Genetics and Molecular Research 15 (3): gmr.15038560 
GAGAGACTCACAAGGC-3' and reverse: 5'-GCTCCACGATGGTGTAGTCCTC-3'; for GAPDH, forward: 5'-GCAAGTTCCACGGCACAG-3' and reverse: 5'-GGTTCACGCCCAT CACAA-3'.

The PCR efficiency of the two primer pairs was within the range $0.8-1.2$; therefore, DsRed expression was determined by the $\Delta \Delta \mathrm{Ct}$ method.

\section{Western blot analysis}

Total protein was extracted from fibroblasts obtained from the ear tips of the six goats and CFC3-KI nuclear donor cells, as well as from the cell lines treated with 5-Az (7 days) and TSA (24 h), using Mammalian Protein Extraction Reagent (Catalog No. CW0002; ComWin Biotech, China). After quantitative analysis with a bicinchoninic acid (BCA) protein kit (Catalog No. 23227; Thermo Scientific), all of the samples were diluted to the same concentration and added to a sodium dodecyl sulfate polyacrylamide gel electrophoresis (SDS-PAGE) loading buffer (Catalog No. CW0027A; ComWin Biotech). The samples were boiled for $5 \mathrm{~min}$ and proteins were separated by SDS-PAGE (12\% gel) prior to transfer to nylon membranes. The membranes were blocked with $5 \%$ skimmed milk powder (Catalog No. 232100; BD Biosciences, USA), and then incubated with an anti-red fluorescent protein (RFP) antibody (1:2000 dilution, Catalog No. Ab62341; Abcam, UK) and an anti-alpha tubulin antibody (1:2000 dilution, Catalog No. Ab125267; Abcam) at $4{ }^{\circ} \mathrm{C}$ overnight. Next, the membranes were incubated with horseradish peroxidase-conjugated goat anti-rabbit IgG H\&L secondary antibody (1:10,000 dilution, Catalog No. Ab6721; Abcam) for $1 \mathrm{~h}$. After washing for $5 \mathrm{~min}$ in tris-buffered saline and Tween 20 to remove redundant secondary antibodies, the membranes were incubated using a SuperSignal ${ }^{\circledR}$ West Pico substrate (Catalog No. 34080; Thermo Scientific). Western blot images were acquired using a Tanon 5200 Automatic Chemiluminescence Imaging Analysis System (Tanon, China).

SDS-PAGE was run under the same experimental conditions for all of the samples (with 6\% SDS stacking gel at $90 \mathrm{~V}$ for $30 \mathrm{~min}$ and $12 \%$ SDS-PAGE separating gel at $120 \mathrm{~V}$ for $60 \mathrm{~min}$ ), and the concentrations of the protein samples were determined by the BCA method and diluted to the same concentration when loading.

\section{Bisulfite sequencing}

Genomic DNA was extracted from fibroblasts (passage P5-P6) obtained from the ear tips of the goats, CFC3-KI cells, and both cell lines treated with 5-Az (7 days) using a DNeasy ${ }^{\circledR}$ Blood and Tissue Kit (Qiagen). The DNA obtained from each sample (180 ng) was subject to bisulfite modification using an EpiXplore ${ }^{\mathrm{TM}}$ Methyl Detection Kit (Catalog No. 631968; Clontech) and dissolved in $20 \mu \mathrm{L}$ elution buffer. Subsequently, $1 \mu \mathrm{L}$ gDNA was used as the template for bisulfite sequencing-PCR (BS-PCR) amplification with EpiTaq ${ }^{\mathrm{TM}} \mathrm{HS}$ (for bisulfite-treated DNA) (Catalog No. R110A; TaKaRa). The PCR primers for BS were designed online using the MethPrimer software (http://www.urogene.org/methprimer/). The sequences of nested PCR primers that were used to improve the accuracy of the BS and the abundance of PCR products were as follows; for the first PCR, forward: 5'-TAGTTATTAATAGTAATTAA-3' and reverse: 5'-AATCTAACGATTCACTAAAC-3'; for the second PCR, forward: 5'-ATTACGGGGTTATTAGTTTA-3' and reverse: 5'-CCAACTCTACTTATATAAAC-3'. The BS-PCR products covered $511 \mathrm{bp}$ of the CMV promoter, including $30 \mathrm{CpG}$ loci, and were

Genetics and Molecular Research 15 (3): gmr.15038560 
purified following separation by $1 \%$ agarose gel electrophoresis. They were then ligated into a T-Vector pMD19 (Simple) (Catalog No. 3271; Takara), thermally transformed into Escherichia coli HST08 Premium Electro-Cells (Catalog No. 9028; Takara), plated, and cultured overnight at $37^{\circ} \mathrm{C}$. At least 10 to 11 positive clones were selected for sequencing (TaKaRa), and the results were aligned with the sequence of the original CMV promoter.

\section{Statistical analysis}

The IBM SPSS software was used for the statistical analysis. All of the data are reported as means $\pm \mathrm{SD}$. A significance of $\mathrm{P}<0.05$ obtained in Pearson correlation analyses indicated statistical significance. For a comparison of the DNA methylation levels, nonparametric comparison tests (Mann-Whitney U-test) were conducted.

\section{RESULTS}

\section{DsRed protein expression in transgenic cashmere goats}

The images in Figure 1, obtained in daylight and following UV excitation in the dark, show obvious differences in DsRed expression in different transgenic individuals. The identities of the transgenic cashmere goats and their corresponding data are shown in Table 1. Red fluorescence was visible in different locations at the whole-body level of three transgenic individuals (ZK110321, ZK110324-2, and ZK110323-3) under both daylight and UV light in the dark, while no fluorescence was observed in individuals ZK0310-1, ZK0311-1, or ZK110324-1.

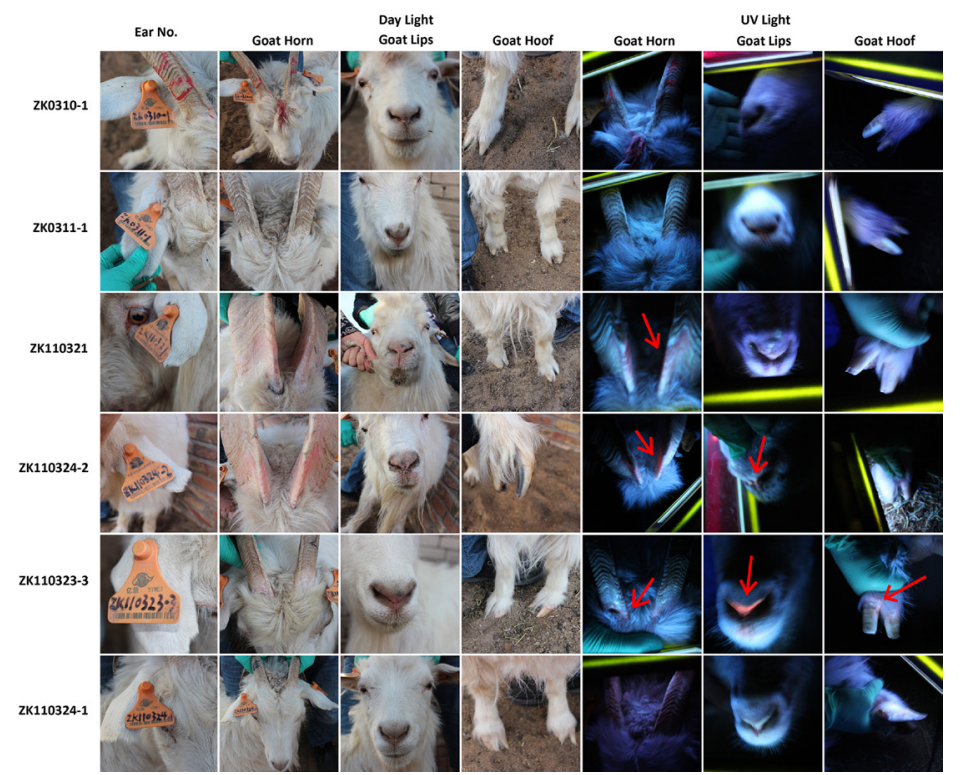

Figure 1. DsRed protein expression in transgenic cashmere goats. Red fluorescence expression images obtained in daylight and following excitation with a portable ultraviolet (UV) source in the dark. Red arrows indicate visible red fluorescence expression.

Genetics and Molecular Research 15 (3): gmr.15038560 
We also observed red fluorescence expression in fibroblasts sampled from the ear tips of the live transgenic cashmere goats, as can be seen in the untreated cell group in Figure 2. We observed obvious red fluorescence in fibroblasts (passage P5-P6) from ZK110321 and ZK110324-2 and transfected nuclear donor CFC3-KI cells (positive control), while little red fluorescence was observed in fibroblasts from ZK0311-1 or ZK110324-1. No red fluorescence was visible in fibroblasts from ZK0310-1, ZK110323-3, or CFC3 cells (negative control).

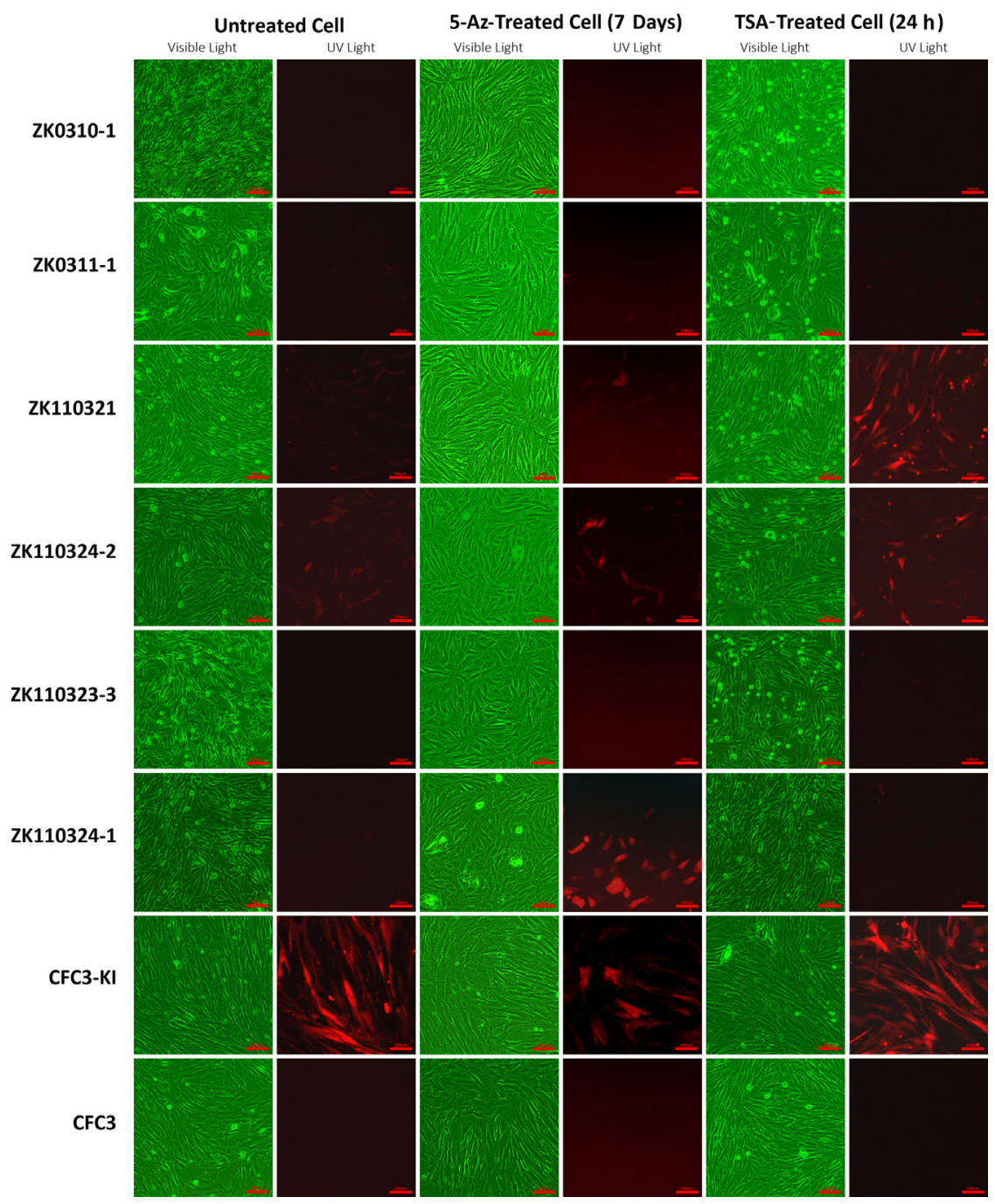

Figure 2. Red fluorescence expression in cultured cells. Red fluorescence expression in fibroblasts sampled from the ear tips of live transgenic cashmere goats, transfected nuclear donor CFC3-KI cells (positive control), and CFC 3 cells (negative control). Cells were treated with 5-azacytidine (5-Az) (7 days) or trichostatin A(TSA) (24 h); untreated cells were used as a control to which the same volume of Dulbecco's phosphate-buffered saline was added. Images were obtained under a fluorescence microscope. Red fluorescence was observed in ZK0311-1,ZK110321, and ZK110324-1 cellsfollowing 5-Aztreatment.AfterTSAtreatment, increasedredfluorescencewas observedinZK110321,ZK1103233, and CFC3-KI cells. No marked changes in red fluorescence were detected in the other cells after drug treatment. 
As shown in Figure 2, the red fluorescence intensity in 5-Az-treated transgenic cells from ZK0311-1, ZK110324-1, and ZK110324-2 was higher than that in untreated transgenic cells, while no obvious changes were detected in cells from ZK0310-1, ZK110321, or ZK110323-3. Red fluorescence intensity was lower in the 5-Az-treated cells than in the untreated CFC3-KI transgenic cells, which exhibited high levels of red fluorescence. Compared with untreated transgenic cells, red fluorescence intensity in TSA-treated ZK110321, ZK110324-2, ZK110323-3, and CFC3- KI transgenic cells increased with more positive cells observed, while no obvious changes were observed in ZK0311-1 or ZK110324-1 cells. Red fluorescence was visible in the treated ZK110323-3 cells, while no fluorescence-positive cells were observed in ZK0310-1 or the transgenic CFC3 cells.

No red fluorescence was visible at the cellular or phenotypic levels in ZK0310-1, while red fluorescence was observed at the phenotypic and cellular levels in ZK110321 and ZK110324-2. Furthermore, red fluorescence expression exhibited an asynchronous trend at the cellular and phenotypic levels in ZK0311-1, ZK110323-3, and ZK110324-1.

\section{Analysis of exogenous gene integration in transgenic cashmere goats}

As shown in Figure 3, the full-length sequence of the complete DsRed expression cassette $C M V p+D s R e d+$ PolyA (CDA full-length sequence, $1499 \mathrm{bp}$ ) was present in the six transgenic cashmere goats and the nuclear donor cells. No mutations were detected after sequencing the purified PCR products, which confirmed that silencing of the exogenous genes in the transgenic cashmere goats was not due to structural changes in the genes.

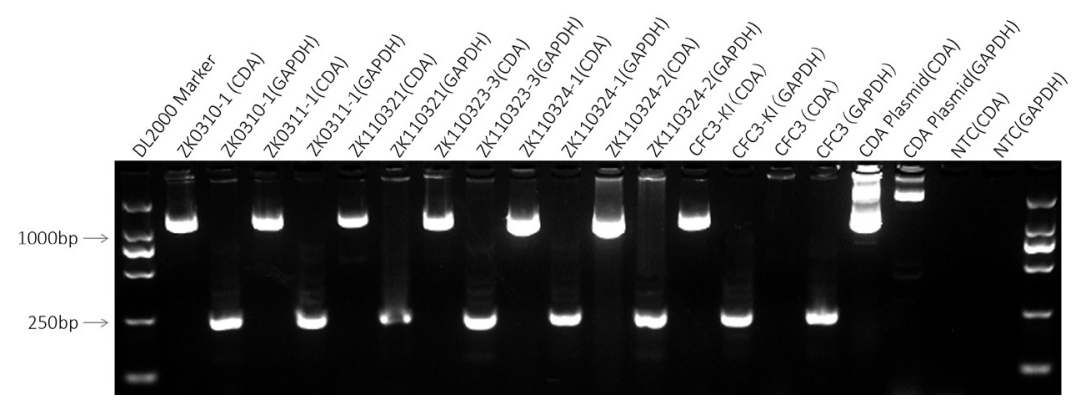

Figure 3. Identification of the DsRed expression cassette in transgenic cashmere goats. The amplified target fragment CDA $(C M V p+D s R e d+$ PolyA $)$ was 1499 bp long and the glyceraldehyde 3-phosphate dehydrogenase (GAPDH) fragment was 249 bp long. The gel was run under the same experimental conditions ( $1 \%$ agarose gel at $120 \mathrm{~V}$ for $30 \mathrm{~min})$.

Two pairs of primers within the CMV promoter and DsRed were used for determining the exogenous gene copy number (Table 2). Differences in the copy numbers of the two fragments were observed between individual goats.

A comparison of the red fluorescence phenotype of the transgenic cashmere goats and DsRed mRNA expression based on its exogenous gene copy number revealed that the presence or absence of red fluorescence was not correlated with the copy number of the exogenous gene ( $C M V p$ fragment, $r=0.541, \mathrm{P}=0.267$; DsRed fragment, $\mathrm{r}=0.322, \mathrm{P}=0.534$ ). This suggests that the exogenous gene copy number does not control the level of expression of the exogenous gene in transgenic cashmere goats.

Genetics and Molecular Research 15 (3): gmr.15038560 
Table 2. Quantitative polymerase chain reaction analysis of the copy number of the exogenous gene ( $C M V p$ and DsRed fragment) in transgenic cashmere goats.

\begin{tabular}{l|c|c|c|c|c|c|c}
\hline Cell No. & ZK110321 & ZK110324-2 & ZK110323-3 & ZK110324-1 & ZK0310-1 & ZK0311-1 & CFC3 \\
\hline Copy number of the CMVp fragment in diploid cells & $3.48 \pm 0.24$ & $3.36 \pm 0.32$ & $1.24 \pm 0.09$ & $1.79 \pm 0.15$ & $2.82 \pm 0.40$ & $0.78 \pm 0.02$ & $0.08 \pm 0.03$ \\
\hline Copy number of the DsRed fragment in diploid cells & $4.48 \pm 0.27$ & $6.28 \pm 0.71$ & $1.32 \pm 0.10$ & $2.51 \pm 0.33$ & $3.77 \pm 0.10$ & $1.93 \pm 0.27$ & $0.01 \pm 0.00$ \\
\hline
\end{tabular}

$\mathrm{N} \geq 5$, correlation between copy number and DsRed mRNA expression: $C M V p$ fragment, $r=0.541, \mathrm{P}=0.267$; DsRed fragment, $r=0.322, \mathrm{P}=0.534$.

\section{Epigenetic modifications in fibroblasts from the ear tips of transgenic cashmere goats}

Investigation of the optimal 5-Az concentration for use in the experiments revealed that it had a strong impact on exogenous gene expression in transgenic cells, with low toxicity observed with the addition of $5 \mu \mathrm{M} 5-\mathrm{Az}$ in the culture medium. Similar investigations of a TSA concentration gradient showed that this effect was achieved with $1 \mu \mathrm{M}$ TSA. Following the treatment of cells with 5-Az (7 days) or TSA $(24 \mathrm{~h})$ at these concentrations, the transgenic cells were evaluated for expression of the endogenous transgene. Red fluorescence was observed following excitation of the untreated and treated cells, and images were obtained in daylight and following excitation with a portable UV source in the dark (Figure 2). Expression of DsRed mRNA was analyzed by real-time fluorescence qPCR (Figure 4), and protein expression was analyzed by western blotting (Figure 5). The methylation level of the CMV promoter before and after 5-Az treatment is shown in Figure 6.

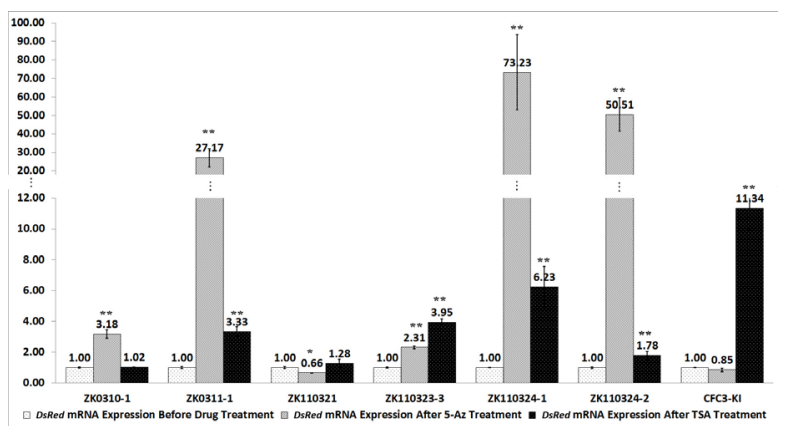

Figure 4. Relative DsRed expression after 5-azacytidine (5-Az)/trichostatin A (TSA) treatment. *Significant difference at $\mathrm{P}<0.05 ; * *$ significant difference at $\mathrm{P}<0.01$.

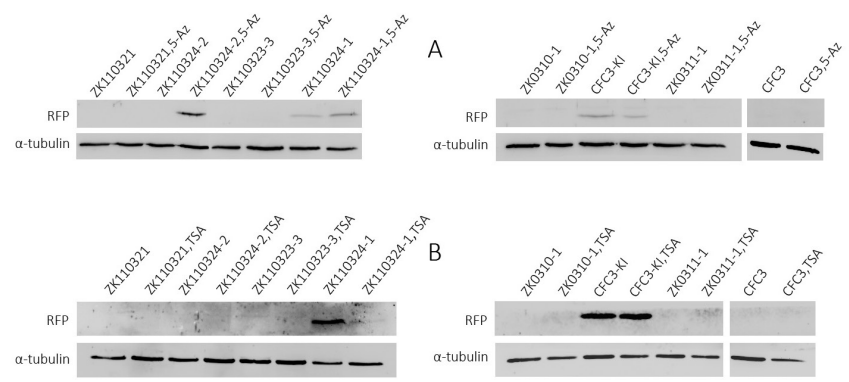

Figure 5. Western blot analysis of DsRed protein expression. Fibroblasts from the ear tips of transgenic cashmere goats were treated with 5-azacytidine (5-Az) (7 days) or trichostatin A (TSA) (24 h). A. Comparison between untreated and 5-Az-treated cells. B. Comparison between untreated and TSA-treated cells. 


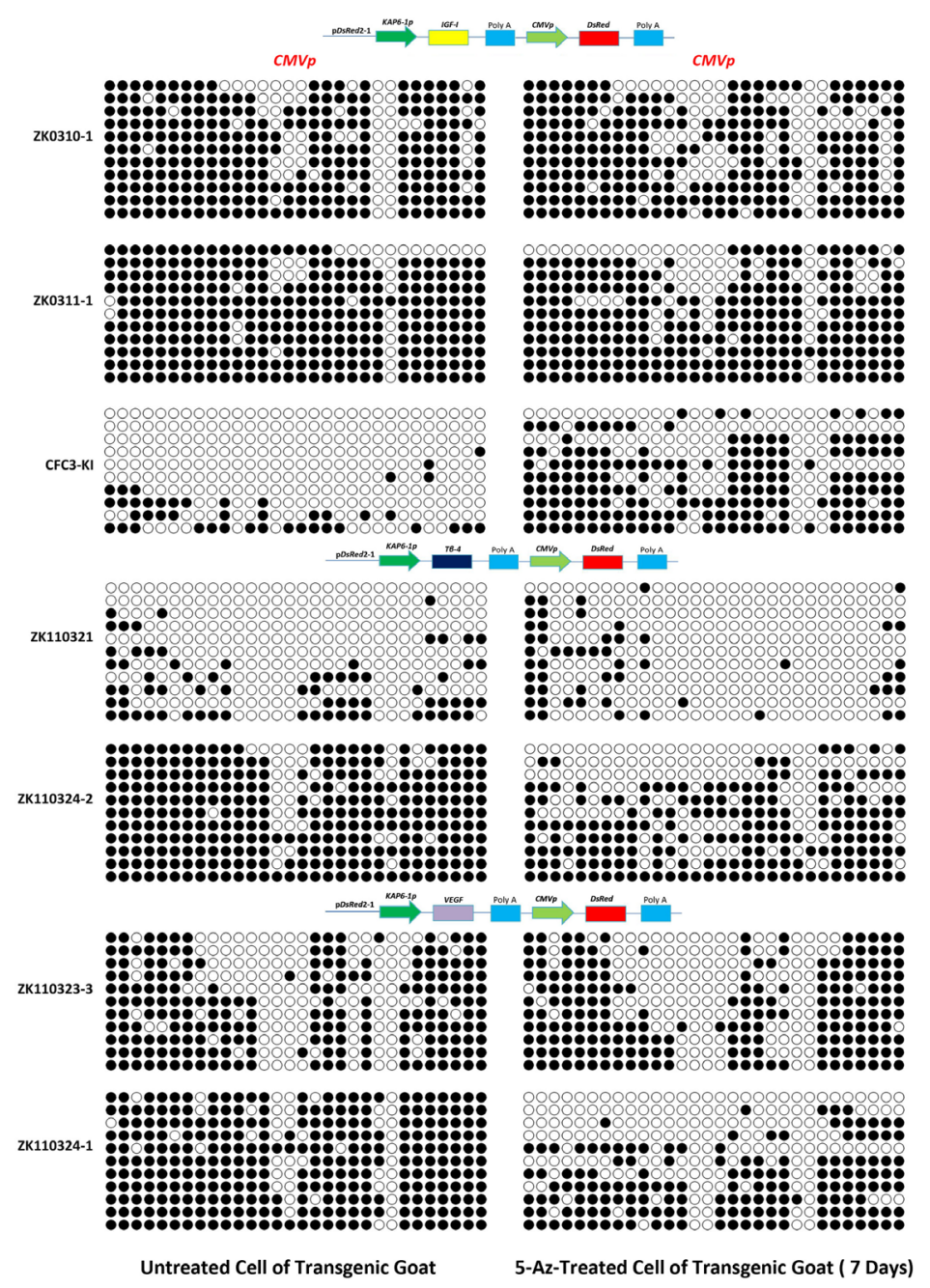

Figure 6. Cytomegalovirus (CMV) promoter methylation status. The black and white dots represent the methylation of $30 \mathrm{CpG}$ sites in a 511-bp fragment of the CMV promoter.

An analysis of covariance revealed that both 5-Az and TSA treatment increased the expression level of DsRed mRNA in fibroblasts of the transgenic cashmere goats, excluding individual differences (different drug treatment, $\mathrm{P}=0.001$; different goat individuals, $\mathrm{P}=0.001$; Table 3 ).

DsRed mRNA expression in the transgenic cashmere goats is shown in Table 3, and the changes in DsRed mRNA and protein expression in all transgenic cells after treatment are shown in Figures 4 and 5, respectively. The results demonstrate that exogenous gene expression in transgenic cashmere goat fibroblasts increased after drug treatment, indicating the potential of this approach for overcoming transgene silencing.

As shown in Figure 5A, no RFP protein was detected in ZK110321, ZK110324-2, ZK110323-3, ZK0310-1, or ZK0311-1 cells before 5-Az treatment. However, RFP protein 
expression significantly increased in 5-Az-treated ZK110324-1 and ZK110324-2 cells, with an obvious RFP-immunoreactive band detected after treatment that was not detected before treatment. In contrast, RFP protein expression decreased in 5-Az-treated CFC3-KI cells. As shown in Figure 5B, no RFP protein was detected in ZK110321, ZK110324-2, ZK110323-3, ZK0310-1, or ZK0311-1 cells before TSA treatment. However, RFP protein expression in untreated ZK110324-1 cells significantly decreased as a result of TSA treatment. No obvious changes were observed in RFP protein expression in the CFC3-KI cells (positive control) following TSA treatment.

\section{Table 3. Test of between-subjects effects.}

\begin{tabular}{l|c|c|c|c|c|c}
\hline Source & Type III sum of squares & d.f. & Mean square & F & P & Partial Eta squared \\
\hline Corrected model & $11,366.771^{\mathrm{a}}$ & 8 & $1,420.846$ & 5.324 & 0.000 & 0.563 \\
\hline Intercept & 5.935 & 1 & 5.935 & 0.022 & 0.882 & 0.001 \\
\hline mRNA expression before drug treatment & 1.545 & 1 & 1.545 & 0.006 & 0.940 & 0.000 \\
\hline Different drug treatment & $3,564.391$ & 1 & $3,564.391$ & 13.357 & 0.001 & 0.288 \\
\hline Different individual & $7,801.476$ & 6 & $1,300.246$ & 4.873 & 0.001 & \\
\hline Error & $8,806.120$ & 33 & 266.852 & & & \\
\hline Total & $27,652.253$ & 42 & & & & \\
\hline Corrected total & $20,172.891$ & 41 & & & & \\
\hline
\end{tabular}

Dependent variable, $D s R e d$ mRNA expression after drug treatment. ${ }^{a} R$ squared $=0.563$ (adjusted $R$ squared $=0.458$ ).

Subsequently, we investigated the methylation status of the CMV promoter in the genomes of transgenic cells treated with 5-Az (Figure 6). As shown in Figure 7, the CMV promoter methylation levels in untreated fibroblasts obtained from the ear tips of ZK0310-1, ZK0311-1, ZK110324-1, ZK110324-2, and ZK110323-3 were as high as 74.4, 84.7, 81.9, 88.2, and 58.7\%, respectively, while lower levels were detected in ZK110321 and CFC3-KI cells (21.2 and 14.3\%, respectively). Furthermore, the methylation level of the CMV promoter was significantly negatively correlated with the relative DsRed mRNA expression $(r=-0.930, \mathrm{P}=0.002)$.

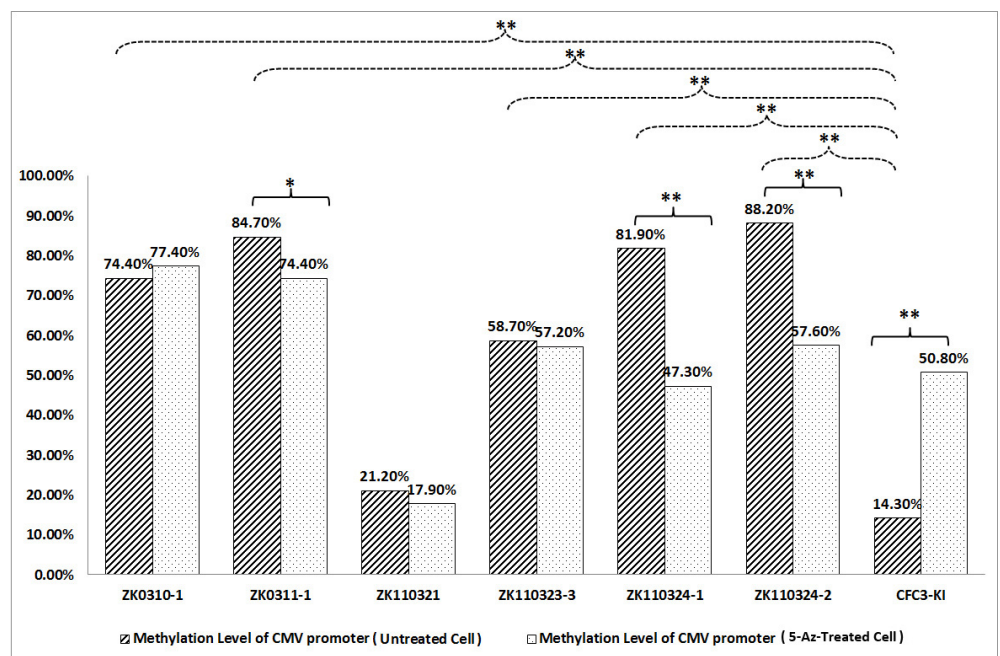

Figure 7. Changes in cytomegalovirus (CMV) promoter methylation levels after 5-azacytidine (5-Az) treatment. *Significant difference at $\mathrm{P}<0.05 ; * *$ significant difference at $\mathrm{P}<0.01$. 
The methylation levels of the CMV promoter after 5-Az treatment are shown in Figure 7. In ZK0311-1, ZK110324-1, ZK110324-2, and CFC3-KI cells, the CMV promoter was highly sensitive to 5-Az treatment with obvious changes observed in its methylation level, while there was an insignificant decrease in CMV methylation level in ZK0310-1, ZK110321, and ZK110323-3 cells.

We also found that CMV methylation levels in the exogenous gene-silenced individuals ZK0310-1, ZK0311-1, ZK110323-3, ZK110324-1, and ZK110324-2 were significantly higher than those in CFC3-KI cells; however, in ZK110321, which had high DsRed expression, there was no significant difference between CMV methylation levels (Figure 7).

Evaluation of DsRed expression in the transgenic cashmere goats revealed synchronized exogenous gene silencing at four levels in ZK0310-1 (mRNA, protein, cell, and whole-body), while gene silencing was asynchronous at the phenotypic and molecular levels in the remaining individuals. For example, the red fluorescence observed in the ZK110323-3 phenotype was absent at the cellular and molecular levels. In contrast, red fluorescence was absent at the phenotypic level in ZK110324-1, but was detected at the cellular and molecular levels. This indicates differences in the degree of silencing of the CMV promoter between different tissues, which suggests that the gene-silencing effect is tissue-specific.

\section{DISCUSSION}

In transgenic animals, the phenomenon of exogenous gene silencing at the phenotypic level is observed in many species. In this study of transgenic cashmere goats, we found obvious exogenous gene silencing in some individuals. Our results indicate that in transgenic cashmere goats generated through the random integration method, the expression efficiency of the same transfected promoter and exogenous gene varies at both the individual phenotypic and molecular level, even to the extent that the red fluorescence phenotype was not observed at the whole-body level in some individuals.

It is necessary to consider the position effect in the context of exogenous gene silencing in transgenic animals generated through the random integration method. However, our findings clearly indicate that the transgenic cashmere goats examined were carrying multiple copies of the transgene in their genome. It is possible that the exogenous gene was spread across various locations in the genomes of the cashmere goats, and that expression of any copy of the gene in the genome could have affected the phenotype at the cellular or whole-body level. Therefore, we suggest that the impact of the position effect in individual transgenic cashmere goats is limited.

In previous studies, the integration copy number was found to be proportional to the exogenous gene expression level in transgenic pigs (Hofmann et al., 2003; Kong et al., 2009); however, we found that the copy number of exogenous genes was variable in transgenic cashmere goats, with most individuals carrying multiple copies. A comparison of copy number and gene expression at the mRNA and protein levels using red fluorescence showed that the copy number of the exogenous gene was not related to its expression in individual goats. It can be speculated that this discrepancy between our results and those reported in transgenic pigs (Kong et al., 2009) is related to species differences or the insertional locus of the transgene. Garrick et al. (1998) found that a reduction in copy number results in a marked increase in transgene expression, and is accompanied by decreased chromatin compaction and methylation at the transgene locus. Furthermore, the presence of multiple homologous copies of a transgene within a concatameric array can have a repressive effect on gene expression in

Genetics and Molecular Research 15 (3): gmr.15038560 
mammalian systems (Garrick et al., 1998). This may explain the phenomenon of high copy number and low DsRed mRNA expression that was observed in the transgenic cashmere goats ZK03101 and ZK110324-1. Differences in the copy numbers of two adjacent fragments might be due to differences in the breakage patterns of the transfected vectors, and differences in the number of vector copies inserted into the genome after separation of the promoter and the transgene.

In addition, the reliability of the quantitative reverse transcription-PCR method when investigating the copy number of exogenous genes in large transgenic livestock was verified; with good reproducibility and controllable error, it should be the first choice to detect the copy number of exogenous genes in transgenic animals.

The high methylation level of the histone H3K9 and DNA in most bovine somatic cell nuclear transfer embryos before implantation was reported as early as 2003 (Santos et al., 2003). Many researchers have also investigated the reprogramming dynamics of epigenetic modifications in nuclear transfer embryos, with abnormal DNA demethylation and methylation events observed in mice (Chung et al., 2003; Mann et al., 2003) and cows (Kang et al., 2002; Enright et al., 2003a). Abnormalities in histone acetylation have also been reported in bovine somatic cell nuclear transfer embryos (Enright et al., 2003a). Incomplete epigenetic reprogramming is also a major cause of abnormal gene expression and subsequent dysplasia in nuclear transfer embryos. Moreover, for those transgenic animals that survive pregnancy and development, exogenous gene silencing is still likely to occur in adults, resulting in transgenic inefficiency, weak or absent target characteristics, and other problems. In previous reports, exogenous gene silencing in transgenic mice (Hofmann et al., 2006) and pigs (Kong et al., 2009) was often attributed to epigenetic modifications after cloning, such as excessive methylation of the exogenous gene promoter and abnormal histone acetylation in the chromosomes of transgenic animals. Therefore, in the somatic cell cloning of mice (Kishigami et al., 2006), pigs (Zhang et al., 2007), rabbits (Shi et al., 2008), and other animals, chemical compounds such as 5-Az and TSA were added to the embryo culture system to promote the reprogramming of reconstructed embryos in order to achieve a higher cloning success rate (Enright et al., 2003b; Rybouchkin et al., 2006).

5-Az affects cells via two mechanisms: at low concentrations, it inhibits the activity of DNA methyltransferase, leading to the hypomethylation of genomic DNA (Cataldo et al., 2009); and, as the chemical analog of cytosine, it may participate in DNA and RNA synthesis at high concentrations, resulting in the covalent binding of 5-Az in the new molecule and subsequent cytotoxicity (Mueller and Florek, 2010). In this study, we investigated the effects of 5-Az-induced hypomethylation on the reprogramming and expression of exogenous genes in transgenic cells.

TSA is an organic compound that selectively inhibits the deacetylation activity of mammalian histones (HDAC I and HDACII), resulting in the maintenance of histone acetylation, which allows transcription factor binding and continued gene activation (Berman et al., 2000). In this study, we used 5-Az and TSA to investigate exogenous gene silencing in transgenic cashmere goat cells, and to explore the correlation between exogenous gene silencing and histone acetylation in transgenic fibroblasts in vitro. Previous reports have also indicated that the methylation status of the CMV promoter is a key factor in the regulation of exogenous gene expression in transgenic animals (Kong et al., 2009). Red fluorescence expression in transgenic cashmere goats has only been evaluated in external tissues and organs, such as the horn, the hoof, and the lip. We found that red fluorescence expression in fibroblasts from ear tips was not completely synchronous with that at the phenotypic level. Previous studies have also found differences in the expression patterns of CMV promoter-driven transgenes at the

Genetics and Molecular Research 15 (3): gmr.15038560 
cellular and individual levels in different transgenic animals (Wakula et al., 2011), and tissue specificity has been reported in transgenic cattle (Yang et al., 2008), sheep (Liu et al., 2013), and other heavy livestock. Bisulfite sequencing of the exogenous CMV promoter in 5-Az-treated and untreated transgenic cells revealed a high methylation status in fibroblasts of four of the six transgenic cashmere goats did not exhibit the external red fluorescence phenotype. Of the six individuals with high CMV methylation at the cellular level, five exhibited very low or no RFP expression. A parallel analysis of DsRed mRNA expression and promoter methylation status revealed a significant negative correlation between the two, which is consistent with reports in mice (Wang et al., 2010) and pigs (Hofmann et al., 2006). This observation suggests that the exogenous gene silencing detected at the mRNA level in transgenic cashmere goats in this study was significantly related to the methylation level of the CMV promoter.

Following 5-Az treatment, changes were observed in DsRed mRNA expression and CMV promoter methylation, although sensitivity to this drug varied among individuals. A correlation analysis revealed a positive correlation between DsRed mRNA expression and the methylation status of the CMV promoter. Based on these experimental results, it can be concluded that methylation inhibitors, such as 5-Az, can rescue exogenous gene silencing in transgenic cells. However, differences were observed in the responses of different cells to the same concentration of 5-Az, and although the methylation level of some transgenic cells was low, the level of DsRed mRNA expression was lower than that of nuclear donor cells. Therefore, high CMV promoter methylation status may not be the only cause of exogenous gene silencing.

TSA treatment of the transgenic cells was performed to explore the effect of changes in histone acetylation on DsRed mRNA expression, and to determine whether abnormalities in the degree of histone acetylation are involved in the mechanism of exogenous gene silencing in transgenic cashmere goats. In this study, DsRed mRNA expression increased in TSA-treated cells, indicating that TSA-mediated inhibition of histone deacetylation can indeed improve the expression of exogenous genes in transgenic cells. Therefore, the exogenous gene silencing detected in individual transgenic cashmere goats was the result of CMV promoter methylation combined with abnormal histone acetylation. However, in ZK110324-1, RFP protein expression in TSA-treated cells significantly decreased, which might have been associated with the biological activity of TSA itself, rather than the downregulation of gene expression caused by the change in the acetylation status of genomic histones.

Transgene expression in fibroblasts was analyzed at both the transcriptional and translational levels. Negative epigenetic modifications of DNA and histones are clearly involved in transgene silencing in cultured fibroblasts. Treatment with TSA, and particularly with 5-Az, reduced the DNA methylation levels of the CMV promoter, leading to upregulation of transcription in three of the six individual goats, and only a slight increase in the transcription level in two of the six cases; therefore, transcription was rescued in most cases. This effect was not surprising, because transcription is under the direct control of epigenetic regulation. However, these treatments were less effective in upregulating the translation of DsRed mRNA. Significantly increased DsRed protein expression was detected by western blot analysis, and red fluorescence was observed in two of the six cases. These observations indicate the potential of TSA and 5-Az treatment to rescue the biological activity of silenced exogenous transgenes in adult-derived transgenic cells under culture conditions.

In this study, we selected the optimal concentrations of 5-Az and TSA for fibroblasts, which are important reference values for how to improve exogenous gene silencing in transgenic animal production. We found that exogenous gene expression varied in individual transgenic 
cashmere goats, with exogenous gene silencing found to be related to the methylation status of the CMV promoter driving the expression of the exogenous gene and abnormal acetylation of the genome. In addition, we found that treatment with the methylation inhibitor 5-Az and the deacetylation enzyme inhibitor TSA altered exogenous gene expression patterns, indicating that both $5-\mathrm{Az}$ and TSA can be used to improve exogenous gene expression in transgenic animals in the future.

\section{Conflicts of interest}

The authors declare no conflicts of interest.

\section{ACKNOWLEDGMENTS}

We are grateful to Kuan Lu and Shaoqing Liu (Yi-Wei White Cashmere Goat Farm, Inner Mongolia Autonomous Region of China) for their help with sample preparation and collection. Research supported by the National Natural Science Foundation of China (\#31101698), the National High Technology Research and Development Program of China (“863” Program) (\#2013AA102506), and the National Natural Science Foundation of China (\#31160228). The funders played no role in the study design, data collection and analysis, decision to publish, or preparation of the manuscript.

\section{REFERENCES}

Berman HM, Westbrook J, Feng Z, Gilliland G, et al. (2000). The Protein Data Bank. Nucleic Acids Res. 28: 235-242. http://dx.doi.org/10.1093/nar/28.1.235

Brooks AR, Harkins RN, Wang P, Qian HS, et al. (2004). Transcriptional silencing is associated with extensive methylation of the CMV promoter following adenoviral gene delivery to muscle. J. Gene Med. 6: 395-404. http:// dx.doi.org/10.1002/jgm. 516

Cataldo VD, Cortes J and Quintas-Cardama A (2009). Azacitidine for the treatment of myelodysplastic syndrome. Expert Rev. Anticancer Ther. 9: 875-884. http://dx.doi.org/10.1586/era.09.61

Chung YG, Ratnam S, Chaillet JR and Latham KE (2003). Abnormal regulation of DNA methyltransferase expression in cloned mouse embryos. Biol. Reprod. 69: 146-153. http://dx.doi.org/10.1095/biolreprod.102.014076

Enright BP, Jeong BS, Yang X and Tian XC (2003a). Epigenetic characteristics of bovine donor cells for nuclear transfer: levels of histone acetylation. Biol. Reprod. 69: 1525-1530. http://dx.doi.org/10.1095/biolreprod.103.019950

Enright BP, Kubota C, Yang X and Tian XC (2003b). Epigenetic characteristics and development of embryos cloned from donor cells treated by trichostatin A or 5-aza-2'-deoxycytidine. Biol. Reprod. 69: 896-901.http://dx.doi.org/10.1095/ biolreprod.103.017954

Garrick D, Fiering S, Martin DI and Whitelaw E (1998). Repeat-induced gene silencing in mammals. Nat. Genet. 18: 5659. http://dx.doi.org/10.1038/ng0198-56

Grassi G, Maccaroni P, Kaiser H, D'Ambrosio E, et al. (2003). Inhibitors of DNA methylation and histone deacetylation activate cytomegalovirus promoter-controlled reporter gene expression in human glioblastoma cell line U87. Carcinogenesis 24: 1625-1635. http://dx.doi.org/10.1093/carcin/bgg118

Guo X, Yang D, Ao X, Wu X, et al. (2009). Production of transgenic cashmere goat embryos expressing red fluorescent protein and containing IGF1 hair-follicle-cell specific expression cassette by somatic cell nuclear transfer. Sci. China Ser. C Life Sci. 52: 390-397. http://dx.doi.org/10.1007/s11427-009-0041-4

Harris PM, McBride BW, Gurnsey MP, Sinclair BR, et al. (1993). Direct infusion of a variant of insulin-like growth factor-I into the skin of sheep and effects on local blood flow, amino acid utilization and cell replication. J. Endocrinol. 139: 463-472. http://dx.doi.org/10.1677/joe.0.1390463

Hofmann A, Kessler B, Ewerling S, Weppert M, et al. (2003). Efficient transgenesis in farm animals by lentiviral vectors. EMBO Rep. 4: 1054-1058.http://dx.doi.org/10.1038/sj.embor.7400007

Genetics and Molecular Research 15 (3): gmr.15038560 
Hofmann A, Kessler B, Ewerling S, Kabermann A, et al. (2006). Epigenetic regulation of lentiviral transgene vectors in a large animal model. Mol. Ther. 13: 59-66.http://dx.doi.org/10.1016/j.ymthe.2005.07.685

Kang YK, Park JS, Koo DB, Choi YH, et al. (2002). Limited demethylation leaves mosaic-type methylation states in cloned bovine pre-implantation embryos. EMBO J. 21: 1092-1100. http://dx.doi.org/10.1093/emboj/21.5.1092

Kishigami S, Mizutani E, Ohta H, Hikichi T, et al. (2006). Significant improvement of mouse cloning technique by treatment with trichostatin A after somatic nuclear transfer. Biochem. Biophys. Res. Commun. 340: 183-189. http:// dx.doi.org/10.1016/j.bbrc.2005.11.164

Kong QR, Wu ML, Huan YJ, Zhang L, et al. (2009). Transgene expression is associated with copy number and cytomegalovirus promoter methylation in transgenic pigs. PLoS One 4: e6679. http://dx.doi.org/10.1371/journal. pone. 0006679

Liu C, Wang L, Li W, Zhang X, et al. (2013). Highly efficient generation of transgenic sheep by lentivirus accompanying the alteration of methylation status. PLoS One 8: e54614.http://dx.doi.org/10.1371/journal.pone.0054614

Mann MR, Chung YG, Nolen LD, Verona RI, et al. (2003). Disruption of imprinted gene methylation and expression in cloned preimplantation stage mouse embryos. Biol. Reprod. 69: 902-914. http://dx.doi.org/10.1095/ biolreprod.103.017293

Mueller A and Florek M (2010). 5-Azacytidine/Azacitidine. In: Small molecules in oncology (Martens UM, ed.). SpringerVerlag, Berlin, pp. 159-170.

Philp D, Nguyen M, Scheremeta B, St-Surin S, et al. (2004). Thymosin beta4 increases hair growth by activation of hair follicle stem cells. FASEB J. 18: 385-387.

Rudolph NS (1999). Biopharmaceutical production in transgenic livestock. Trends Biotechnol. 17: 367-374. http://dx.doi. org/10.1016/S0167-7799(99)01341-4

Rybouchkin A, Kato Y and Tsunoda Y (2006). Role of histone acetylation in reprogramming of somatic nuclei following nuclear transfer. Biol. Reprod. 74: 1083-1089. http://dx.doi.org/10.1095/biolreprod.105.047456

Santos F, Zakhartchenko V, Stojkovic M, Peters A, et al. (2003). Epigenetic marking correlates with developmental potential in cloned bovine preimplantation embryos. Curr. Biol. 13: 1116-1121. http://dx.doi.org/10.1016/S09609822(03)00419-6

Shi LH, Ai JS, Ouyang YC, Huang JC, et al. (2008). Trichostatin A and nuclear reprogramming of cloned rabbit embryos. J. Anim. Sci. 86: 1106-1113. http://dx.doi.org/10.2527/jas.2007-0718

Teschendorf C, Warrington KH, Siemann DW and Muzyczka N (2002). Comparison of the EF-1 alpha and the CMV promoter for engineering stable tumor cell lines using recombinant adeno-associated virus. Anticancer Res. 22: 3325-3330.

Wakula P, Bisping E, Kockskamper J, Post H, et al. (2011). CMV promoter is inadequate for expression of mutant human RyR2 in transgenic rabbits. J. Pharmacol. Toxicol. Methods 63: 180-185. http://dx.doi.org/10.1016/j. vascn.2010.09.007

Wang Y, Song YT, Liu Q, Liu C, et al. (2010). Quantitative analysis of lentiviral transgene expression in mice over seven generations. Transgenic Res. 19: 775-784. http://dx.doi.org/10.1007/s11248-009-9355-Z

Yamamoto S, Konishi I, Tsuruta Y, Nanbu K, et al. (1997). Expression of vascular endothelial growth factor (VEGF) during folliculogenesis and corpus luteum formation in the human ovary. Gynecol. Endocrinol. 11: 371-381. http:// dx.doi.org/10.3109/09513599709152564

Yang PH, Wang JW, Gong GC, Sun XZ, et al. (2008). Cattle mammary bioreactor generated by a novel procedure of transgenic cloning for large-scale production of functional human lactoferrin. PLoS One 3: e3453. http://dx.doi. org/10.1371/journal.pone. 0003453

Yuan JL, Jin Y, Zhu B, Wang W, et al. (2012). Somatic cell nuclear transfer efficiency associated with donor cell types in the cashmere goat. J. Anim. Vet. Adv. 11: 4578-4584.

Zhang Y, Li J, Villemoes K, Pedersen AM, et al. (2007). An epigenetic modifier results in improved in vitro blastocyst production after somatic cell nuclear transfer. Cloning Stem Cells 9: 357-363. http://dx.doi.org/10.1089/clo.2006.0090

Genetics and Molecular Research 15 (3): gmr.15038560 